Classification

Physics Abstracts

$07.35+\mathrm{k}-73.20 \mathrm{Dx}-79.80+\mathrm{w}$

\title{
Transverse magnetic field effects on the resonant tunneling current
}

\author{
L. A. Cury ( ${ }^{1}$, A. Celeste $\left({ }^{1}\right)$, B. Goutiers $\left({ }^{1}\right)$, J. C. Portal $\left({ }^{1}\right)$, D. L. Sivco $\left({ }^{2}\right)$ and \\ A. Y. Cho $\left({ }^{2}\right)$ \\ (1) INSA-CNRS, Avenue de Rangueil, 31077 Toulouse Cedex, France et SNCI-CNRS, Avenue \\ des Martyrs, 38048 Grenoble Cedex, France \\ (2) AT \& T Bell Laboratories, Murray Hill, New Jersey 07974, U.S.A.
}

(Received 30 May 1990, revised 18 June 1990, accepted 11 July 1990)

\begin{abstract}
Résumé. - Un système à double barrière à semiconducteurs basé sur les alliages (InGa)As(InAl)As est étudié sous un fort champ magnétique transverse $B_{\perp}$ (perpendiculaire au courant). On étudie plus particulièrement la variation expérimentale de la tension $V_{\mathrm{p}}$ associèe au courant pic en fonction du champ magnétique $B_{\perp}$. A très fort champ, le comportement attendu de $V_{p}$ en $B_{\perp}^{2}$ est observé. Dans le domaine des bas champs magnétiques une déviation du comportement quadratique est mise en évidence. Le comportement plus compliqué de $V_{\mathrm{p}}$ en fonction de $B_{\perp}$ est obtenu au travers d'un lissage numérique. Nous proposons une discussion qualitative, de ces résultats, fondée sur notre travail antérieur (L. A. Cury, A. Celeste, J. C. Portal, Solid-States Electron. 32 (1989) 1689) ainsi qu'une comparaison avec les résultats d'autres auteurs.
\end{abstract}

Abstract. - Resonant tunneling process in an (InGa)As-(InAl)As symmetric double-barrier
structure subjected to a transverse magnetic field $B_{\perp}$ (perpendicular to the current) is
investigated: We particularly focus on the experimental behaviour of $V_{\mathrm{p}}$, the voltage at the
current peak position, as a function of the magnetic field $B_{\perp}$. For strong magnetic fields a clear
dependence on $B_{\perp}^{2}$ is observed, as expected. However, an original result is obtained at small
magnetic fields where a deviation from the parabolic behaviour is observed. The more
complicated dependence on $B_{\perp}$ of $V_{\mathrm{p}}$ is obtained from a numerical fit. A qualitative discussion is
proposed on the basis of a previous publication (L. A. Cury, A. Celeste, J. C. Portal, Solid-States
Electron. 32 (1989) 1689) and the differences with the results of other authors are pointed out.

(InGa)As-(InAl)As double-barrier devices [1-4] have recently attracted considerable interests. High peak to valley ratios have been reported recently [5] on double-barrier diodes based on (InGa)As-(InAl)As lattice matched to InP and even better results were found in strained layer structures [6]. The presence in this system of low effective masses in both (InAl)As-barrier [7] and (InGa)As-well [8], high conduction band discontinuities [9] and conduction band non-parabolicity [10] makes it appropriate to the study of vertical transport.

In this letter, we analyse the transverse magnetic field effects on the current-voltage characteristics (Fig, 1). In figure 2 we show the experimental behaviour of the voltage at the current peak position $V_{\mathrm{p}}$ as a function of the applied transverse magnetic field $B_{\perp}$. At the 


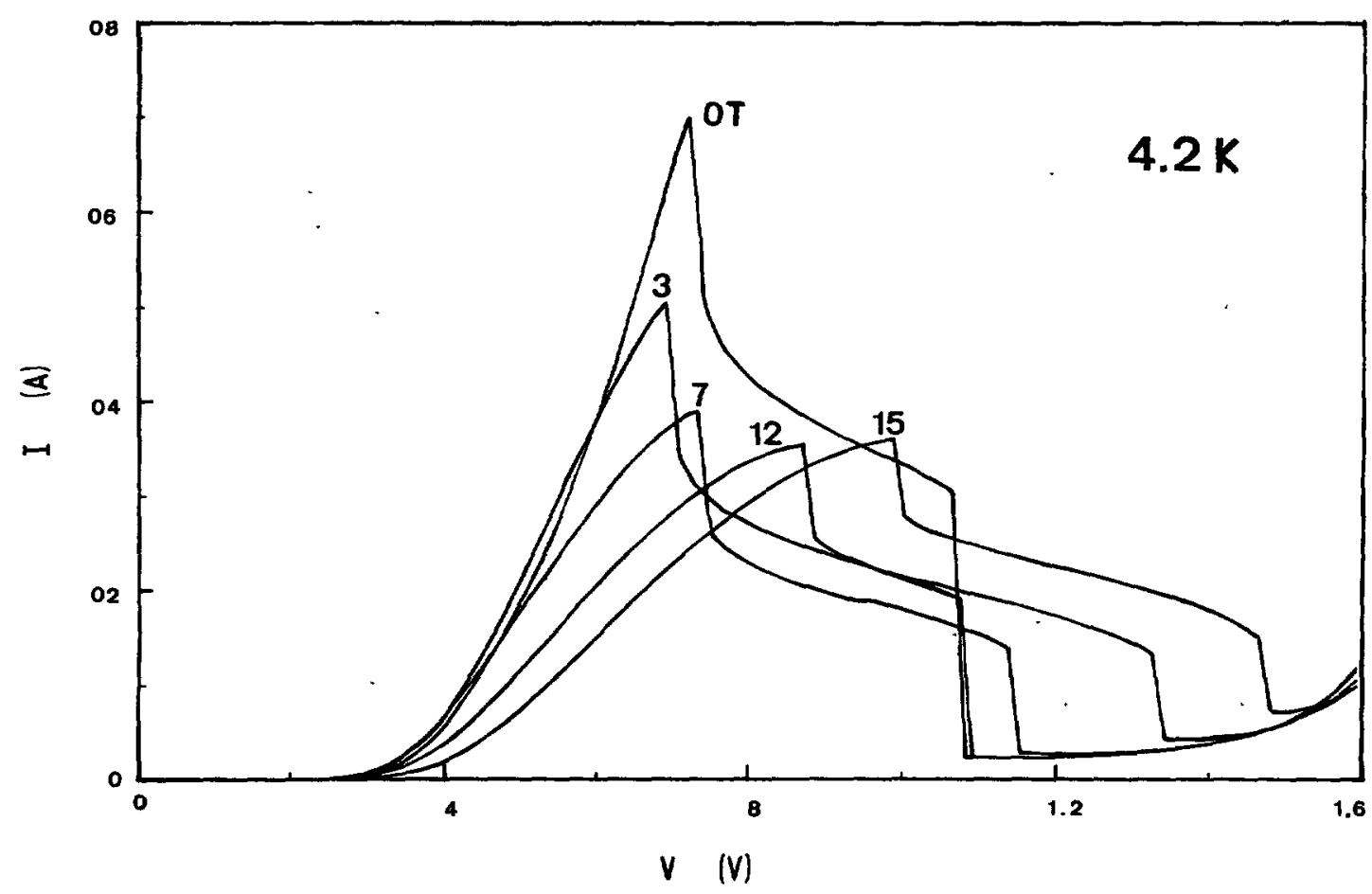

Fig. 1. - I versus $V$ characteristics for several transverse magnetic fields.

high magnetic fields the expected $B_{\perp}^{2}$ behaviour is obtained [11] whereas at low magnetic fields, a more dependence on $B_{\perp}$ is observed in agreement with our previous theoretical work (Ref. [14]). A numerical fit of the experimental $V_{\mathrm{p}}$ versus $B_{\perp}$ curve (Fig. 2a), reveals a polynomial behaviour up to the fourth order in $B_{\perp}$. The terms proportional to $B_{\perp}^{3}$ and $B_{\perp}^{4}$ are the adjustment terms which we believe indicate the non-parabolicity effects in our structure. A comparative analysis with other experiments is done. We conclude that the behaviour at small magnetic fields is mainly connected with the effective Fermi energy $E_{\mathrm{fe}}$ in the region adjacent to the emitter barrier.

The sample studied has two $-50 \AA$ with (InAl)As barrier and the enclosed $50 \AA$ width (InGa)As well between them. The complete structure, in order of growth from the $\mathbf{n}^{+}-\operatorname{In} P$ (100) substrate is : (i) $0.5 \mu \mathrm{m}$ of $n=1 \times 10^{18} \mathrm{~cm}^{-3} \mathrm{In}_{1-y} \mathrm{Ga}_{y} \mathrm{As}$; (ii) $25 . \AA$ of undoped in $\mathrm{In}_{1-y} \mathrm{Ga}_{y} \mathrm{As}$ spacer layer ; (iii) $50 \AA$ of undoped $\mathrm{In}_{1-} \mathrm{Al}_{x} \mathrm{As}$ barrier; (iv) $50 \AA$ of residually doped $n=8 \times 10^{15} \mathrm{~cm}^{-3} \mathrm{In}_{1-y} \mathrm{Ga}_{y}$ As well ; (v) $50 \AA$ of undoped $\mathrm{In}_{1-x} \mathrm{Al}{ }_{x} \mathrm{As}$ barrier ; (vi) $25 \AA$ of undoped $\mathrm{In}_{1-y} \mathrm{Ga}_{y}$ As spacer layer; (vii) $0.5 \mu \mathrm{m}$ of $n=1 \times 10^{18} \mathrm{~cm}^{-3} \mathrm{In}_{1-y} \mathrm{Ga}_{y} \mathrm{As}$ top contact. The $\mathrm{Al}$ and $\mathrm{Ga}$ concentrations are $x=0.48$ and $y=0.47$, respectively, $50 \mu \mathrm{m}$ mesas were fabricated using standard lithography techniques.

Our current-voltage characteristics are shown in figure 1 corresponding to several transverse magnetic fields. The following features are observed when $\mathbf{B}_{\perp}$ is increased : (i) the current peak is shifted to high voltages; (ii) the negative differential resistance region broadens; (iii) the magnitude of the peak current $I_{\mathrm{p}}$ is first decreased at small $B_{\perp}$, and increases at higher magnetic fields. These effects have been observed by Ben Amor and colleagues [2] and they are well explained qualitatively in the respective reference. The main difference between the previous results and ours, is that we observe a singular behaviour 

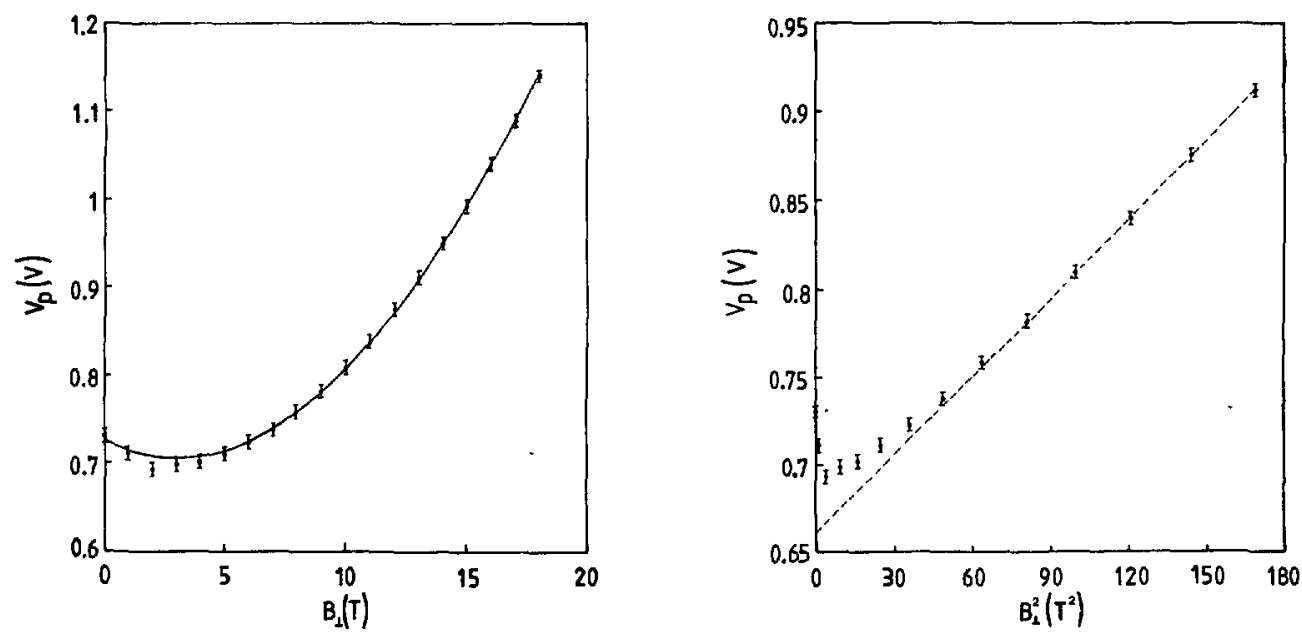

Fig. 2. - a) $V_{\mathrm{p}}$ versus $B_{\perp}$ curve. The full line is the numerical fit obtained from equation (1); b) $V_{\mathrm{p}}$ versus $B_{\perp}^{2}$ curve. The linear behaviour is observed for $B_{\perp}>8 \mathrm{~T}$. For $B_{\perp} \leqslant 8 \mathrm{~T}$ the deviation of the linear behaviour is clearly verified. The dashed line is a guide for the eyes.

at small magnetic fields (Figs. $2 \mathrm{a}$ and $2 \mathrm{~b}$ ) which was not observed before. The following of this paper attempts to give a qualitative explanation of this original effect.

For comparison we will analyse some results obtained by Ben Amor and colleagues in reference [2] and [12] where the two magnetic field geometries $\mathbf{B} \perp \mathbf{J}$ and $\mathbf{B} \| \mathbf{J}$ are studied, respectively. The same sample studied in these references, has a large $400 \AA$ (residually doped $\left.10^{15} \mathrm{~cm}^{-3}\right)$ spacer layer separating the wide $(0.5 \mu \mathrm{m})$ very doped $\left(n=2 \times 10^{18} \mathrm{~cm}^{-3}\right)$ $\mathrm{In}_{0.53} \mathrm{Ga}_{047} \mathrm{As}$ layer to the barrier. Calculations of the Fermi energy considering nonparabolicity corrections to first order [13] give $E_{\mathrm{f}}=122 \mathrm{meV}$ in their very doped (InGa)As contact layer $\left(n=2 \times 10^{18} \mathrm{~cm}^{-3}\right)$ at $4.2 \mathrm{~K}$. The equilibrium condition between the Fermi energies guaranties that the conduction band mininum in the spacer layer region will roughly have an energy $E=\frac{1}{2} E_{\mathrm{g} \text { InGas }}+E_{\mathrm{f}}$ above the conduction band minimum in the doped region. $E_{\mathrm{g}}$ is the energy gap in the (InGa)As bulk layer. Under bias, the spacer layer will give rise to a roughly triangular quantum barrier separating the Fermi sea to the accumulation layer and associated quasi-two dimensional electron gas (2DEG) in the region adjacent to the emitter barrier.

The magnetoquantum oscillations in the current for $\mathbf{B} \| \mathbf{J}$ observed in reference [12] revealed the presence of one electric subband in the accumulation layer. The effective Fermi energy $E_{\mathrm{fe}}$ in this region, relative to this electric subband, can be calculated by $E_{\mathrm{fe}}=\hbar e B_{\mathrm{f}} / m . B_{\mathrm{f}}$ is the fundamental field defined from the periodicity of the oscillations. In reference [12] $B_{\mathrm{f}}$ has a linear behaviour from $12 \mathrm{~T}$ up to $33 \mathrm{~T}$ corresponding to voltages above the threshold for resonant tunneling. A saturation is present around $15 \mathrm{~T}$, slightly below $V_{\mathrm{p}}$. At $V_{\mathrm{p}}$, the fundamental field abruptly increases to $18.6 \mathrm{~T}$. An estimate, using $m=0.041 m_{\mathrm{e}}[8]$ and $B_{\mathrm{f}}=18.6 T$, gives $E_{\mathrm{fe}} \simeq 52 \mathrm{meV}$ at the peak voltage which is smaller than the Fermi energy in their heavily doped contact region $(122 \mathrm{meV})$.

Our sample has a very thin $(25 \AA)$ spacer layer. Under bias no accumulation layer is formed. The effective Fermi energy corresponding to the doped region $(0.5 \mu \mathrm{m}$ of $n=1 \times 10^{18} \mathrm{~cm}^{-3} \operatorname{In}_{0.53} \mathrm{Ga}_{0.47} \mathrm{As}$ ) is $80 \mathrm{meV}$ [13] sensibly higher than in the Ben Amor's sample. The resonant tunneling process implies directly $3 \mathrm{D}$-electrons of the Fermi sea in the 
emitter. For magnetic fields smaller than $8 \mathrm{~T}$ (Figs. 2a) a minimum is observed for a non-zero magnetic field position indicating that the voltage $V_{\mathrm{p}}$ has a more complex behaviour than the $B_{\perp}^{2}$ one in this range of fields. This effect has not been observed in precedent works [2, 11]. In our previous paper [14], it was predicted that a large enough Fermi energy is necessary to observe a deviation of the quadratic behaviour of $V_{\mathrm{p}}$ with $B_{\perp}$. Our present results seem to be in a good qualitative agreement with this statement, since our sample presents a higher effective Fermi energy necessary to observe this effect.

Ben Amor and colleagues in reference [2] have not studied directly the behaviour of $V_{\mathrm{p}}$ as a function of $B_{\perp}$. They claimed that this behaviour is comparable to that of the turn-off voltage which is defined as the bias at which the valley current reaches an absolute minimum. Their $V_{\text {off }}$ versus $B_{\perp}$ curve does not show the same behaviour at small magnetic fields than ours in figure $2 \mathrm{a}$. We believe this is caused by the lower value of the effective Fermi energy in the $2 \mathrm{D}$ accumulation layer of their structure.

A deviation of the $B_{\perp}^{2}$ behaviour, as observed in figure $2 \mathrm{~b}$ at small magnetic fields, was interpreted in the previous work [14] by an increase of the coefficient of the term in $B_{\perp}$ contained in the $V_{\mathrm{p}}$ expression. At small fields and if the effective Fermi energy in the region adjacent to the emitter barrier $\left(E_{\mathrm{fe}}\right)$ is large enough, the $K_{y}$ electron wave-vector contained in this coefficient could take values such that the term in $B_{\perp}$ could become more important than the $B_{\perp}^{2}$ one.

A larger dependence on $K_{y}$ wave-vector for a larger $E_{\mathrm{fe}}$ which increases the term proportional to $B_{\perp}$, as interpreted in our previous work (Ref. [14]), is reinforced by our numerical fit (Fig. 2a) where the experimental points are well described by the fourth order polynome

$$
V_{\mathrm{p}}=0.7260-0.01347 B_{\perp}+0.0022 B_{\perp}^{2}+0.0000001 B_{\perp}^{3}-0.0000005 B_{\perp}^{4} .
$$

As we can see in equation (1), the coefficient on $B_{\perp}$ is about 6 times larger than the $B_{\perp}^{2}$ one in absolute value. At small magnetic fields the term on $B_{\perp}$ must predominate. This is in a good agreement with the qualitative discussion in reference [14]. However, the dependence on $B_{\perp}^{3}$ and on $B_{\perp}^{4}$ has not been predicted. The coefficients of these terms in equation (1) are much smaller in comparison with the coefficients on $B_{\perp}$ and on $B_{\perp}^{2}$ but they turn out to be important for larger magnetic fields. We believe that these terms can come from the conduction band non-parabolicity, which is stronger in the (InGa)As(InAl)As structures and that was not taken into account in the previous work (Ref. [14]).

The formalism developped by Ekenberg [15], based on the conduction band dispersion in the bulk of a direct-gap III- $V$ semiconductors expanded up to fourth order in $K$, seems to be a common way to introduce the non-parabolicity effects in the envelope function method as well as the effects of the transverse magnetic field. However, it is not trivial from an analytical point of view. We believe this is a possible solution to explain our experimental fourth order polynomial behaviour of $V_{\mathrm{p}}$ as a function of $\boldsymbol{B}_{\perp}$. This will be the subject of a future work.

In summary, we have investigated the current versus voltage characteristics as a function of the transverse magnetic field $B_{\perp}$. We have observed a deviation from the expected effect on the voltage $V_{\mathrm{p}}$ at the current peak position, in the range of small magnetic fields, that had not been observed in precedent works. We associate our results to the growth parameters of our sample where a large enough effective Fermi energy is present in the region adjacent to the emitter barrier. This is in a good qualitative agreement with reference [14], where the deviation from the linear behaviour of $V_{\mathrm{p}}$ versus $B_{\perp}^{2}$ has been predicted in this case. Our experimental results reinforce the statement in reference [14] that the $K_{y}$ wave-vector of the incident electron is an important factor to establish the behaviour of $V_{\mathrm{p}}$ as a function of $B_{\perp}$. 
Although our results give a good qualitative agreement with the predictions in reference [14], a more complete theory, including conduction band non-parabolicity corrections, is necessary to explain analytically the fourth order polynomial behaviour of $V_{\mathrm{p}}$ versus $B_{\perp}$ obtained from our numerical fit.

\section{Acknowledgments.}

One of us (L.A.C.) is grateful to Conselho Nacional de Pesquisa - CNPq Brasil for financial support. We acknowledge the financial support from Conseil Regional de Midi-Pyrenees and European Communities. We are also grateful to the LAAS (Toulouse) for processing the samples.

\section{References}

[1] Capasso Federico, Sen Susanta, Cho Alfred Y., Sivco Deborah L., Appl. Phys. Lett. 53 (1988) 1056.

[2] Amor S. Ben, Rascol J. J. L., Martin K. P., Higgins R. J., Potter R. C., Lakhani A. A., Hier H., Phys. Rev. B 41 (1990) 7860.

[3] Celeste A., Cury L. A., Portal J. C., Allovon M., Maude D. K., Eaves L., Davies M., Heath M., Maldonado M., Solid-States Electron. 32 (1989) 1191.

[4] Payling C. A., White C. R. H., Eaves L., Alves E. S., Leadbeater M. L., Portal J. C., Hodson P. D., Robins D. J., Wallis R. H., Davis J. I., Marshall A. C., Superlat. and Microstruc. 6 (1989) 193.

[5] Muto Shunichi, Inata Tsuguo, SugIYama Yoshihiro, Nakata Yoshiaki, FujI Toshio, Ohnishi Hiroaki, HiYamizu Satoshi, Jpn. J. Appl. Phys. 26 (1987) L220.

[6] Inata Tsuguo, Muto Shunichi, Sasa Shigehiko, Fuji Toshio, Hiyamizu Satoshi, Jpn. J. Appl. Phys. 26 (1987) L1332.

[7] Olego D., Chang T. Y., Silberg E., Caridi E. A., Pinczuk A., Appl. Phys. Lett. 41 (1982) 476.

[8] Alavi K., Aggarwal R. L., Groves S. H., Phys. Rev. B 21 (1980) 1311.

[9] People R., Wecht K. W., Alavi K., Cho A. Y., Appl. Phys. Lett. 43 (1983) 118.

[10] Adelabu J. S. A., Ridley B. K., Davies G. J., Semicond. Sci. Technol. 4 (1989) 677.

[11] Amor S. Ben, Martin K. P., Rascol J. J. L., Higgins R. J., Torabi A., Harris H. M., Summers C. J., Appl. Phys. Lett. 53 (1988) 2540.

[12] Amor S. Ben, Martin K. P., Rascol J. J. L., Higgins R. J., Potter R. C., Lakhani A. A., HIER H., Appl. Phys. Lett. 54 (1989) 1908.

[13] Numerical calculation of $E_{\mathrm{f}}$ at $4.2 \mathrm{~K}$ based on the three-dimensional electronic density

$$
n=\int_{0}^{\infty} g\left(\alpha^{\prime}, \varepsilon\right) f\left(\varepsilon, E_{\mathrm{f}}\right) \mathrm{d} \varepsilon
$$

where $f\left(\varepsilon, E_{\mathrm{f}}\right)$ is the Fermi function, $g\left(\alpha^{\prime}, \varepsilon\right)=\frac{\sqrt{2} m^{3 / 2}}{\pi^{2} h^{3}}\left(1+2 \alpha^{\prime} \varepsilon\right) \sqrt{\varepsilon\left(1+\alpha^{\prime} \varepsilon\right)}$ is the density of levels. $\alpha_{(\mathrm{InGa}) \mathrm{As}}^{\prime}=1.243(1 / \mathrm{eV})^{(10)}$ is the non-parabolicity coefficient.

[14] Cury L. A., Celeste A., Portal J. C., Solid-States Electron. 32 (1989) 1689.

[15] Ekenberg U., Phys. Rev. B 40 (1989) 7714. 